\title{
Sulfoquinovose is a select nutrient of prominent bacteria and a source of hydrogen sulfide in the human gut
}

\author{
Buck T. Hanson ${ }^{1,2} \cdot$ K. Dimitri Kits ${ }^{1} \cdot$ Jessica Löffler ${ }^{1}$ Anna G. Burrichter ${ }^{3,4} \cdot$ Alexander Fiedler $^{3} \cdot$ Karin Denger $^{3}$.

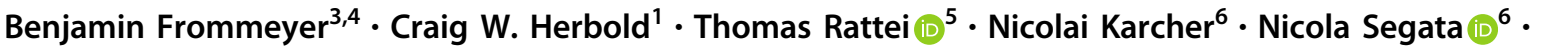 \\ David Schleheck $\mathbb{D}^{3,4} \cdot$ Alexander Loy $\mathbb{D}^{1,7}$
}

Received: 11 September 2020 / Revised: 1 March 2021 / Accepted: 17 March 2021 / Published online: 31 March 2021

(c) The Author(s) 2021. This article is published with open access

\begin{abstract}
Responses of the microbiota to diet are highly personalized but mechanistically not well understood because many metabolic capabilities and interactions of human gut microorganisms are unknown. Here we show that sulfoquinovose (SQ), a sulfonated monosaccharide omnipresent in green vegetables, is a selective yet relevant substrate for few but ubiquitous bacteria in the human gut. In human feces and in defined co-culture, Eubacterium rectale and Bilophila wadsworthia used recently identified pathways to cooperatively catabolize SQ with 2,3-dihydroxypropane-1-sulfonate as a transient intermediate to hydrogen sulfide $\left(\mathrm{H}_{2} \mathrm{~S}\right)$, a key intestinal metabolite with disparate effects on host health. SQ-degradation capability is encoded in almost half of $E$. rectale genomes but otherwise sparsely distributed among microbial species in the human intestine. However, re-analysis of fecal metatranscriptome datasets of four human cohorts showed that SQ degradation (mostly from E. rectale and Faecalibacterium prausnitzii) and $\mathrm{H}_{2} \mathrm{~S}$ production (mostly from B. wadsworthia) pathways were expressed abundantly across various health states, demonstrating that these microbial functions are core attributes of the human gut. The discovery of green-diet-derived SQ as an exclusive microbial nutrient and an additional source of $\mathrm{H}_{2} \mathrm{~S}$ in the human gut highlights the role of individual dietary compounds and organosulfur metabolism on microbial activity and has implications for precision editing of the gut microbiota by dietary and prebiotic interventions.
\end{abstract}

\section{Introduction}

Dietary habits largely modulate the highly personalized composition and temporal dynamics of the human intestinal microbiota and influence disease risk [1-3]. Each individual daily ingests thousands of different

Supplementary information The online version contains supplementary material available at https://doi.org/10.1038/s41396021-00968-0.

David Schleheck

david.schleheck@uni-konstanz.de

$\triangle$ Alexander Loy

alexander.loy@univie.ac.at

1 Division of Microbial Ecology, Centre for Microbiology and Environmental Systems Science, University of Vienna, Vienna, Austria

2 FFoQSI GmbH, Austrian Competence Centre for Feed and Food Quality Safety \& Innovation, Tulln, Austria dietary compounds [4] of which some, such as starch or glucose, are substrates for many microorganisms while others, such as the seaweed polysaccharide porphyran, are select energy sources of metabolically specialized microorganisms [5, 6]. Disentangling the effects of single dietary compounds on gut microbiota membership and activity is important for understanding diet-microbiota interaction mechanisms [7] but complicated because the genetic and physiological capabilities of microorganisms are insufficiently established [8].

3 Department of Biology, University of Konstanz, Konstanz, Germany

4 Konstanz Research School Chemical Biology, University of Konstanz, Konstanz, Germany

5 Division of Computational Systems Biology, Centre for Microbiology and Environmental Systems Science, University of Vienna, Vienna, Austria

6 CIBIO Department, University of Trento, Trento, Italy

7 Joint Microbiome Facility of the Medical University of Vienna and the University of Vienna, Vienna, Austria 
Here, we explored for the first time microbial metabolism of sulfoquinovose (6-deoxy-6-sulfoglucose, SQ) in the human gut. SQ is the polar head group of sulfoquinovosyl diacylglycerol (SQDG), a ubiquitous sulfolipid in the photosynthetic membranes of all land plants, algae, dinoflagellates, and cyanobacteria. SQDG is one of the most abundant organic sulfur compounds in the biosphere and can represent greater than $25 \%$ of the total lipids in common dietary, leafy green vegetables such as spinach, lettuce, and green onion [9, 10]. Studies on the catabolism of dietary SQDG by animals are scarce; in guinea pigs SQDG is deacylated via host-derived lipases to sulfoquinovosyl glycerol (SQG) [11]. It is not known whether host tissues are able to metabolize SQG or SQ. Some Proteobacteria can catabolize SQDG/SQG and SQ analogously to the Embden-Meyerhof-Parnas (EMP) and the Entner-Doudoroff (ED) glycolytic pathways, hence, either via the sulfo-EMP (e.g., commensal and pathogenic Escherichia coli) or the sulfo-ED pathway (e.g., Pseudomonas putida) [12-14]. Recently, a third pathway for SQ degradation via a novel 6-deoxy-6-sulfofructose transaldolase (SFT) was described in the aerobic Bacillus aryabhattai isolate SOS1 [15] and Bacillus megaterium DSM 1804 [16]. The intestinal strains of the Firmicutes species Enterococcus gilvus, Clostridium symbiosum, and Eubacterium rectale also expressed SFT pathway genes during anaerobic, fermentative growth with SQ [15]. Bacterial SQ-degradation products are the excreted $\mathrm{C}_{3}$-organosulfonates 2,3-dihydroxypropane-1sulfonate (DHPS) or 3-sulfolactate, which can serve as sources of sulfite via diverse catabolic pathways of specialized sulfite-respiring and $\mathrm{H}_{2} \mathrm{~S}$-producing Desulfovibrionaceae species $[17,18]$. Although mammalian cells also produce $\mathrm{H}_{2} \mathrm{~S}$ in the cytoplasm and in mitochondria [19], bacteria that anaerobically respire sulfite derived from sulfate or taurine are considered the main sources of $\mathrm{H}_{2} \mathrm{~S}$ in the intestine [20,21]. Because SQDG is a frequent compound in a vegetarian diet and some gut bacteria have the catabolic potential for complete degradation of SQ to $\mathrm{H}_{2} \mathrm{~S}$ via interspecies cross-feeding of 3-sulfolactate or DHPS, SQ could be a so far overlooked green-dietderived source of $\mathrm{H}_{2} \mathrm{~S}$ in the gut. $\mathrm{H}_{2} \mathrm{~S}$ is a Janus-faced metabolite that exerts its manifold beneficial and harmful impacts on the host in a dose-dependent manner [22]. For example, $\mathrm{H}_{2} \mathrm{~S}$ is a mitochondrial energy source, a cellular antioxidant, and an important signaling molecule in mammalian physiology [23]. In contrast, at higher concentrations, $\mathrm{H}_{2} \mathrm{~S}$ can chemically deteriorate the mucosal barrier of the gastrointestinal tract [24] and decrease ATP production through inhibition of cytochrome $c$, potentially leading to cell death [25]. Diets high in saturated fat trigger increased excretion of taurocholic bile acids and promote intestinal expansion of the taurine-utilizing pathobiont Bilophila wadsworthia, exacerbating intestinal inflammation in a susceptible host [26]. Clearly, a more complete knowledge of the sources and sinks of $\mathrm{H}_{2} \mathrm{~S}$ is required to understand what regulates its homeostasis in the gut and whether it is beneficial or detrimental to the host [20, 27].

In this study, we hypothesized that SQ is selectively degraded by specialized bacteria and contributes to the formation of $\mathrm{H}_{2} \mathrm{~S}$ in the human gut. By targeted physiological experiments with human fecal microcosms and monoand co-cultures and by re-analysis of large metagenome and metatranscriptome datasets from human fecal samples, we reveal the identity of bacteria involved in complete degradation of SQ to $\mathrm{H}_{2} \mathrm{~S}$, the activity of the underlying pathways, and provide evidence for their importance for the microbial processes in the gut.

\section{Materials and methods}

Supplementary Materials and Methods provide further details on the methods described below.

\section{Chemicals}

SQ and 3-sulfolactate were synthesized by MCAT GmbH (Donaueschingen, Germany). DHPS was synthesized and validated by NMR and HPLC-MS as reported previously [12]. Taurine, isethionate and all other purchased, routine chemicals were of at least puriss p.a. grade if not otherwise stated.

\section{Human fecal microcosms}

Anaerobic microcosms containing human vegetarian fecal samples were used to evaluate SQ metabolism by the gut microorganisms. Microcosms were subsampled over 10 days for metabolite quantification and community composition analyses.

\section{Pure culture and defined co-culture experiments}

E. rectale DSM 17629 and $B$. wadsworthia strain 3.1 .6 were grown on SQ and DHPS, respectively, as described previously $[15,17,28]$. At intervals, cultures were subsampled for metabolite quantification. B. wadsworthia 3.1.6 cells were harvested from the late exponential growth phase for differential proteomics when grown with the four different organosulfonates or for preparation of anoxic cellfree extracts for DHPS sulfite-lyase enzyme tests. For cocultivation experiments, $E$. rectale DSM 17629 was inoculated first $(n=3)$ and after $81 \mathrm{~h} \mathrm{~B}$. wadsworthia 3.1.6 was co-inoculated. 


\section{Anoxic cell-free extracts and enzyme tests}

DHPS sulfite-lyase assays using cell-free B. wadsworthia 3.1.6 extracts were done as described previously [28] with colorimetric quantification of sulfite and HPLC-UV analysis of derivatizatized hydroxyacetone.

\section{Differential proteomics}

Proteomic analyses of cell-free B. wadsworthia 3.1.6 extracts were done as previously described [28].

\section{Metabolite analyses}

$\mathrm{H}_{2} \mathrm{~S}$ was quantified colorimetrically [29]. Short-chain fatty acids were quantified using capillary electrophoresis. SQ, DHPS, 3-sulfolactate, taurine, and isethionate were quantified as described previously [12]. Hydroxyacetone, shortchain fatty acids, and alcohols in culture experiments were analyzed by an HPLC method described previously [17]. Sulfite in enzyme reactions was quantified using a colorimetric assay (Fuchsin assay) or by derivatization with $N-(9-$ acridinyl)maleimide and HPLC-UV [28].

\section{Fluorescence cell counting}

FISH probes targeting $E$. rectale and $B$. wadsworthia were designed using ARB [30] with optimal hybridization conditions determined on pure cultures of E. rectale DSM 17629 and B. wadsworthia DSM 11045 (Table S1). Aliquots of PFA-fixed microcosm biomass were filtered onto black polycarbonate filters, stained with FISH probes, and counted using standard procedures [31].

\section{Single-cell stable-isotope probing by FISH-Raman microspectroscopy}

PFA-fixed cells from microcosms incubated with $10 \mathrm{mM}$ $\mathrm{SQ}$ and $50 \% \mathrm{D}_{2} \mathrm{O}$ were subjected to liquid-based FISH [32]. Samples were spotted onto an aluminum-coated glass slide and after air drying fluorescence microscopy was used to identify cells for Raman analyses. Single-cell Raman spectra were acquired using a LabRAM HR800 confocal Raman microscope (Horiba Jobin-Yvon) and D-labeling (\% CD) was quantified from integrated C-D and C-H peak areas as previously described [32].

\section{Extraction of nucleic acids}

DNA for amplicon and metagenome sequencing and RNA for metatranscriptome sequencing were extracted following standard procedures [33] that included bead beating for $30 \mathrm{~s}$ on dry ice using a Lysing Matrix E tube (MP Biomedicals).
Purified nucleic acids were stored in $30 \mu \mathrm{l}$ DNase/RNasefree $\mathrm{H}_{2} \mathrm{O}$ at $-80{ }^{\circ} \mathrm{C}$.

\section{S rRNA gene and dsrB amplicon sequencing}

Established two-step PCR barcoding protocols were used for amplicon sequencing of the 16S rRNA gene [34] and $d s r B$ [35]. Barcoded libraries were pooled at equivalent copy numbers $\left(20 \times 10^{9}\right)$ for 300 bp paired-end sequencing on a MiSeq sequencer (Illumina). Sequencing results were analyzed according to the procedures outlined previously $[34,35]$.

\section{Metagenomics}

Samples from three time points $(28,73$, and $114 \mathrm{~h})$ from SQ-amended microcosms were chosen for $150 \mathrm{bp}$ pairedend metagenome HiSeq 3000/4000 (Illumina) sequencing. Trimmed and error-corrected reads were assembled using metaSPAdes [36], and metagenome-assembled genomes (MAGs) were binned using MetaBAT [37]. MAGs were assessed for quality using checkM [38], de-replicated using drep [39], and assigned taxonomy through alignment and phylogenetic placement of concatenated marker genes into the Genome Taxonomy Database reference tree (GTDB-Tk) [40]. Average nucleotide identity comparisons between genomes were calculated using FastANI [41].

\section{Metatranscriptomics}

Metatranscriptome libraries were prepared from three SQamended microcosm time points $(6,20$, and $52 \mathrm{~h})$. Following DNase digestion, rRNA was removed and 1-100 ng was used in each library preparation for multiplexed Illumina RNASeq analysis. Sequencing (50 bp, single-end) was performed using HiSeq 3000/4000 (Illumina). Trimmed reads were mapped to the genomes of E. rectale ATCC 33656 and $B$. wadsworthia 3.1.6, because these genomes are complete and have a very high ANI to the respective E. rectale and $B$. wadsworthia MAGs obtained from the microcosms. Feature counting and differential expression analysis was done using featureCounts within the Subread package [42] and the exactTest function (edgeR), respectively.

\section{Construction of a bacterial YihQ/SftG profile hidden markov-model (HMM)}

Bacterial genomes and MAGs in Genbank as of July 27, 2017 ( $N=103411)$ were screened using hmmsearch [43] for genes that contained the glycoside hydrolase $(\mathrm{GH})$ family 31 pfam (PF01055.25). The resulting dataset $(N=$ $127,608)$ was screened using a YihQ/SftG-specific motif 
that we constructed using signature residues reported by Speciale et al. [44]. A manually curated alignment was used to construct an HMM using hmmbuild [43] (File S1).

\section{Genome analyses and comparative genomics}

All contigs and MAGs from the SQ incubations were screened for known SQ metabolism genes using blastp and the YihQ/SftG HMM. In addition, we searched the NCBI genome database using blastp for organisms that contain the four essential pathway genes-the sulfoquinovosidase (YihQ/SftG), SQ isomerase (SftI), 6-deoxy-6-sulfofructose transaldolase (SftT), and the reductase (SftR) (Table S2). The presence of the SFT gene cluster in 73,798 high-quality MAGs from publicly available human gut metagenomes [45] was searched using a two-step approach (Table S3). First, translated open reading frames were queried with the YihQ/SftG HMM. Second, blast-based searches for SftI, SftT, and SftR were performed against all translated open reading frames of $\mathrm{YihQ} / \mathrm{SftG}$-containing genomes. Genes enabling taurine (tpa), DHPS ( $h p s G H, h p s O, h p s N$, dphA), 3-sulfolactaldehyde (slaB), 3-sulfolactate (suyAB, slsC, $\operatorname{com} C$ ), sulfoacetaldehyde (xsc, sarD), and isethionate (isl $A B)$ catabolism and sulfite reduction $(\operatorname{ds} A B C)$ were identified in 16 representative $d s r A B$-containing human gut bacteria by blastp screening.

\section{Metatranscriptome analysis of publicly available datasets}

Reads from 1,090 paired gut metagenomes/metatranscriptomes were trimmed followed by taxonomic and functional profiling using HUMAnN2 [46] and MetaPh1An2 [47]. Contributions of SFT pathway-encoding organisms to SFT pathway expression were assessed by mapping of the trimmed reads from the 1,090 metatranscriptomes to the SFT pathway-encoding genomes identified in our NCBI search. A similar approach was used to assess expression of $h p s G H$, islAB, and $d s r A B C$ genes in the 16 representative $d s r A B$-containing human gut bacteria (see previous section). The relative abundance of the SFT pathway in the 1,090 metatranscriptomes was evaluated using HUMANn2. Proportional contributions of each organism to total expression of the SFT pathway was calculated as a ratio between the RPKM value for the organism of interest divided by the total RPKM value for the pathway in that sample.

\section{E. rectale transcription network analysis}

Trimmed reads (processed as described above) from the 1090 gut metatranscriptomes were mapped to the E. rectale ATCC 33656 genome. Samples with greater than 5/8 of all
E. rectale ATCC 33656 genes with at least 1 read mapped $(n=244)$ were selected for network construction. A read count-normalized matrix was then used as input into SPIEC-EASI [48]. Significant correlations between gene pairs (positive or negative interaction strength of $>0.099$ ) with any functional prediction $(n=1349$ unique genes, 2326 gene pairs) were then imported into Cytoscape [49] for network visualization. Significant clusters were identified using ClusterONE [50].

\section{Phylogenetic and phylogenomic tree construction}

Phylogenomic analysis of the 93 MAGs from the fecal incubations was performed with GToTree [51] using 74 bacterial single-copy genes. Comparative phylogenetic analysis of the GH family 31 was done by downloading the seed alignment from the pfam database, aligning the sequences using MAFFT [52], and then adding 219 putative novel YihQ/SftG sequences ( 70 characterized GH family 31 proteins, 70 hits from the Uniprot database using the YihQ/ SftG HMM, and 79 unique YihQ/SftG homologs from the 83 genomes encoding the SFT pathway) and 8,696 GH family 31 sequences. For comparative phylogenetic analysis of pyruvate formate-lyase like enzymes, we retrieved amino acid sequences of known choline trimethylamine-lyases, 4hydroxyphenylacetate decarboxylases, hydroxyproline dehydratases, aryl- alkyl-succinate synthases, glycerol dehydratases, and pyruvate formate-lyases from the KEGG ligand enzyme nomenclature database and clustered them at 90\% identity using usearch [53]. Twenty-three glycyl radical enzyme sequences, including known isethionate sulfite-lyases, from genomes of human-gut Desulfovibrionaceae were added to the 1204 reference amino acid sequences. Approximate maximum likelihood trees were constructed from alignments with FastTree2 using the JTT + CAT model.

\section{Results and discussion}

\section{SQ is cooperatively metabolized in fimo mainly by $E$. rectale and $B$. wadsworthia to acetate and $\mathrm{H}_{2} \mathrm{~S}$}

To investigate SQ degradation by human gut microbiota, we constructed triplicate anoxic microcosms with fecal slurries mixed from eight vegetarians and incubated them with SQ (10 mM) or control substrates (Fig. S1A). SQ was completely consumed within $20 \mathrm{~h}$ concomitant with a transient accumulation of DHPS (up to $5 \mathrm{mM}$ ) between 20 and $52 \mathrm{~h}$ (Fig. 1A). As DHPS was consumed, we observed increased production of $\mathrm{H}_{2} \mathrm{~S}$ with a peak at $96 \mathrm{~h}$. In addition, formate accumulated transiently with a peak at $73 \mathrm{~h}$ (about $3 \mathrm{mM}$ ), while acetate gradually increased over $144 \mathrm{~h}$ 
A Metabolites in SQ-amended microcosms

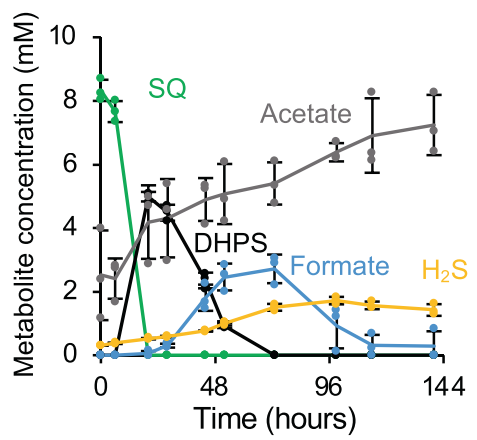

B

Metabolites in unamended microcosms

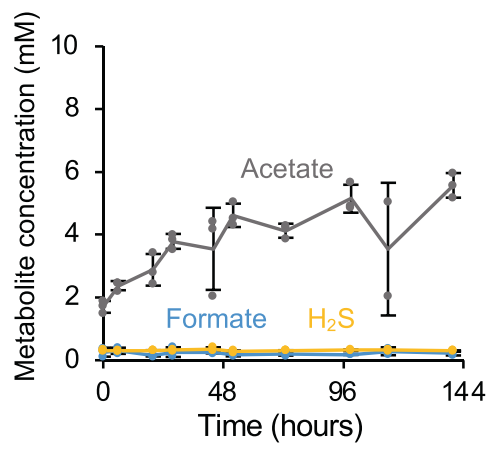

C

16S rRNA gene relative adundance in SQ-amended and unamended microcosms

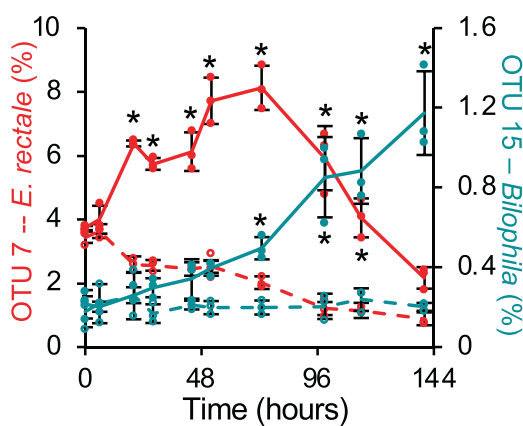

D FISH-positive cells out of DAPI-stained cells in SQ-amended microcosms

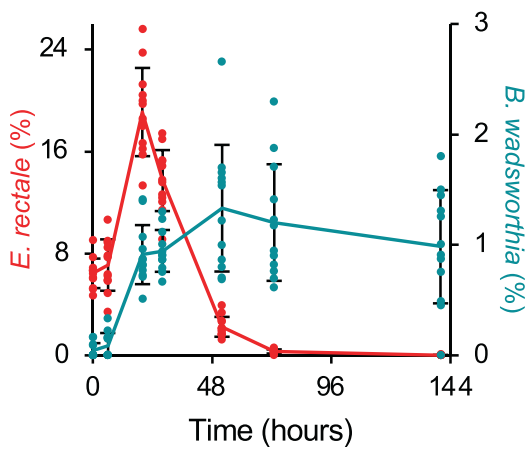

E FISH-D2O Raman microspectroscopy in SQ-amended microcosms

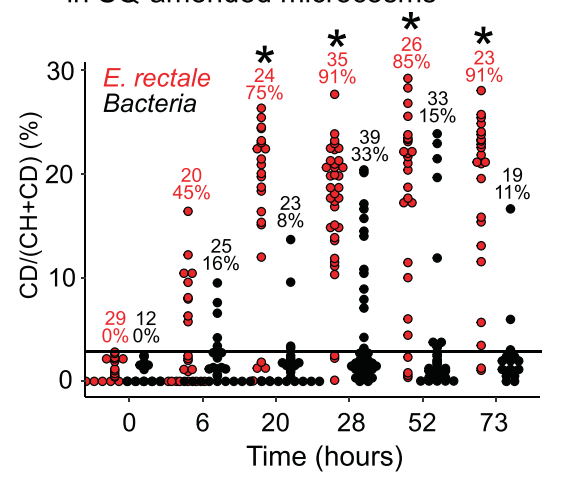

F Sequential co-culture

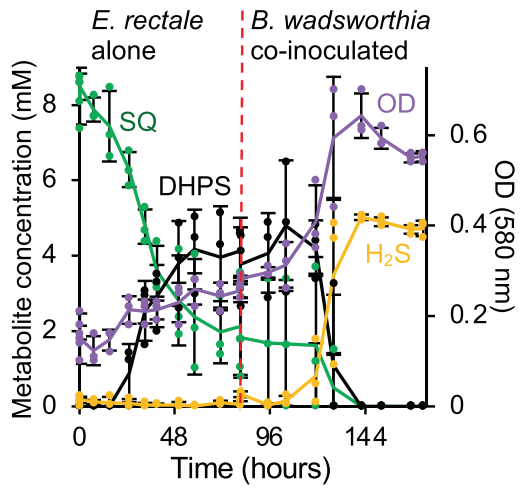

Fig. 1 Sulfoquinovose is anaerobically degraded to $\mathrm{H}_{2} \mathrm{~S}$ by DHPScross-feeding between Eubacterium rectale and Bilophila wadsworthia. A Degradation of SQ (green) in anoxic human fecal microcosms resulted in transient production of DHPS (black) and formate (blue) and accumulation of $\mathrm{H}_{2} \mathrm{~S}$ (yellow) and acetate (gray). Formate is a possible electron donor for respiration of DHPS-derived sulfite. B Unamended control microcosms did not produce DHPS, formate, or $\mathrm{H}_{2} \mathrm{~S}$. C Relative abundance of E. rectale (red) and B. wadsworthia (teal) 16S rRNA gene OTUs in microcosms with SQ (solid lines) and without amendment (dashed lines). D FISH quantification of $E$. rectale and B. wadsworthia in SQ microcosms. Error bars represent one standard deviation of averages from 12 microscopic fields. E FISHRaman analysis of single-cell activity (\%CD labeling) of E. rectale (red) and Bacteria (black) in SQ-amended fecal microcosms

(to $7.5 \mathrm{mM}$ ) (Fig. 1A), compared to unamended control microcosms (Fig. 1B). Primary SQ and secondary DHPS degradation was accompanied by select changes in community composition, with only nine species-level $16 \mathrm{~S}$ rRNA gene operational taxonomic units (OTUs) increasing significantly $(P<0.01)$ in relative abundance compared to unamended microcosms (Fig. S2A, Tables S4 and S5). Temporal abundance changes of the two most strongly increasing OTUs, identified as E. rectale (Agathobacter rectalis [40]) and Bilophila wadsworthia (Fig. 1C, Fig. S2A), were confirmed by fluorescence probe-based microscopy cell counting (Fig. 1D) and corresponded with the consumption of SQ and DHPS, respectively (Fig. 1A, C). Stable isotope probing of individual cells in SQ- and containing $50 \% \mathrm{D}_{2} \mathrm{O}$. Numbers indicate the number of cells analyzed and percentages of active cells (above a $3.54 \% \mathrm{CD}$ background threshold; black bar) and asterisks indicate that FISH-positive E. rectale cells are significantly enriched in $\mathrm{CD}$ vs. $0 \mathrm{~h}(P<0.01)$. F Two-step degradation of SQ via DHPS to $\mathrm{H}_{2} \mathrm{~S}$ by a co-culture of $E$. rectale DSM 17629 (grown in pure culture for $81 \mathrm{~h}$ ) and $B$. wadsworthia 3.1.6 (co-inoculated at $81 \mathrm{~h}$, dotted line). In $\mathbf{A}, \mathbf{B}$, and $\mathbf{C}$, lines represent averages of triplicate measures with error bars representing one standard deviation. Asterisks show significant differences $(P<$ 0.01 ) in OTU relative abundance between matched time points of SQ and unamended microcosms. SQ sulfoquinovose, DHPS 2,3dihydroxypropane-1-sulfonate, OTU operational taxonomic unit, FISH fluorescence in situ hybridization, DAPI 4',6-diamidino-2phenylindole, OD optical density at $580 \mathrm{~nm}$.

heavy water $\left(\mathrm{D}_{2} \mathrm{O}\right)$-amended microcosms demonstrated high cellular activity (up to $29 \%$ D-labeling) of E. rectale (Fig. 1E), suggesting that SQ was utilized for growth [32]. Amplicon sequencing of $d s r B$, encoding the beta-subunit of a key enzyme for $\mathrm{H}_{2} \mathrm{~S}$ production, dissimilatory sulfite reductase, revealed a significant increase in a Bilophila OTU at $20 \mathrm{~h}$ and later $(>44 \mathrm{~h})$ also in two Desulfovibrio OTUs $(P<0.01$; Fig. S1I, Tables S6 and S7), in concert with DHPS and $\mathrm{H}_{2} \mathrm{~S}$ dynamics (Fig. 1A). Consistently, phylogenomic analysis of 93 MAGs from the SQ-amended microcosm (Fig. 1A and Table S8) identified one MAG as E. rectale (SQ_MAG_41, average nucleotide identity (ANI) of $>97 \%$ to 42 E. rectale strains; Tables S8 and S9) and a second MAG as B. wadsworthia (SQ_MAG_14, ANI 
of $>97 \%$ to three B. wadsworthia strains; Tables S8 and $\mathrm{S} 10)$. While a few other bacteria are likely also involved (Fig. S2A), these data suggest that SQ is cooperatively metabolized in the microcosms mainly by E. rectale and B. wadsworthia with net production of acetate and $\mathrm{H}_{2} \mathrm{~S}$. We confirmed this finding by co-culturing isolates of $E$. rectale (DSM 17629) and B. wadsworthia (strain 3.1.6) with SQ. E. rectale grew by fermenting SQ and produced DHPS, which was rapidly consumed after co-inoculation of $B$. wadsworthia leading to near-stoichiometric production of $\mathrm{H}_{2} \mathrm{~S}$ (Fig. 1F). The co-culture reproduced the sulfur metabolite dynamics observed in our SQ-amended microcosms and affirms DHPS-cross-feeding between E. rectale, one of the most abundant gut microbiota members in healthy individuals [54, 55], and B. wadsworthia, a pathobiont associated with gastrointestinal inflammation and cancer [21, 26, 56, 57].

We next applied genome-resolved metatranscriptomics on the fecal microcosms and differential proteomics on pure-culture experiments to identify the genes/proteins involved in complete catabolism of SQ to $\mathrm{H}_{2} \mathrm{~S}$.

\section{E. rectale ferments $S Q$ in the gut via the transaldolase pathway to produce DHPS}

E. rectale SQ_MAG_41 was the only MAG from the SQamended microcosms metagenome that contained a cluster with SQ metabolism genes and $y i h Q / s f t G$ (Fig. 2A), which is co-located with all previously characterized SQdegradation gene clusters [12, 13, 15] and encodes a sulfoquinovosidase (alpha-glucosidase) that cleaves SQ from SQDG/SQG [14, 44]. All sulfoquinovosidase homologs form a separate monophyletic lineage within the GH family 31 tree (Fig. S3). Transcription of the E. rectale yihQ/sftG as well as of 10 flanking genes was significantly upregulated upon SQ degradation in the microcosms $(P<0.02$, Fig. 2B, Table S11). We independently confirmed co-expression of these 11 co-localized genes by constructing a gene expression network for $E$. rectale using publicly available metatranscriptomes from 1090 human fecal samples [58-60] (Fig. S4). Besides yihQ/sftG, this cluster includes all genes of the SFT pathway that was active during SQ fermentation by E. rectale strain DSM 17629 [15]. Here, an SQ isomerase (SftI) converts SQ to 6-deoxy-6sulfofructose. A transaldolase (SftT) then catalyzes interconversion of 6-deoxy-6-sulfofructose with glyceraldehyde-3-phosphate to 3-sulfolactaldehyde and fructose-6-phosphate. Fructose-6-phosphate is funneled into glycolysis for fermentation and growth. 3Sulfolactaldehyde is reduced to DHPS by an NADHdependent reductase (SftR) as an additional fermentation step [15], and the DHPS is exported via SftE.
We subsequently queried 73,793 high-quality MAGs, assembled from 9,428 human gut metagenomes [45], for the four core catalytic enzyme genes (yihQ/sftG, sftI, sftT, sftR) and found a surprisingly narrow distribution of the SFT pathway across human gut species. All four genes were present in only $23(0.5 \%)$ of 4,930 species-level genome bins [45] and mostly at low frequency (Fig. 2A). A notable exception is the $E$. rectale genome bin with $44 \%$ of its 2,301 MAGs encoding the SFT pathway (Fig. 2A), suggesting that $E$. rectale is the most prevalent SQmetabolizing organism in the human gut.

\section{B. wadsworthia desulfonates DHPS to sulfite via an oxygen-sensitive, glycyl radical DHPS sulfite-lyase}

At the start of our study, DHPS was an unknown substrate for B. wadsworthia to generate $\mathrm{H}_{2} \mathrm{~S}$ (Fig. S5A) and we thus sought to identify the genes responsible for DHPS catabolism, such as genes for enzymes converting DHPS to 3-sulfolactate, the substrate for the desulfonating enzyme SuyAB [17]. Screening the genomes of human gut-associated Desulfovibrionaceae for known genes involved in the metabolism of DHPS and other organosulfonates for respiration of sulfite (Fig. S5B) $[17,28]$ revealed that the currently described genes for linking DHPS catabolism to the 3-sulfolactate desulfonation pathways (the DHPS dehydrogenase genes hps $O$ and $d h p A)$ [17, 61] are absent in all known Bilophila genomes, indicating that DHPS might not be metabolized via 3-sulfolactate in $B$. wadsworthia. Instead, we identified for the pure culture of B. wadsworthia 3.1.6 through differential proteomics that it strongly and specifically expresses a four-gene cluster during growth with DHPS. This cluster includes genes for a new glycyl radical DHPS sulfite-lyase system (HpsGH) (Fig. 2C, D, Fig. S5C-E) that was recently identified and characterized [18]. HpsG is a closely related but phylogenetically distinct paralog of isethionate (2-sulfoethanol) sulfitelyase IslA [28] (also designated IseG [62]) (60.6\% identity to HpsG) in B. wadsworthia (Fig. S6). IslA catalyzes isethionate desulfonation, a key reaction in taurine degradation [28, 62]. $\mathrm{HpsH}$ is the activator of HpsG [18], analogous to the IslA-activating, radical SAM enzyme IslB [28]. Accordingly, hps $G$ and $h p s H$ were the most differentially expressed B. wadsworthia genes in the SQ-degrading fecal microcosms (n-fold change of 83 and 93 , respectively; $P=3.8 \times 10^{-8}$ and $1.0 \times 10^{-5}$, respectively, Fig. 2B, Table S12). Highly oxygen-sensitive glycyl radical enzymes are a diverse and abundant protein family of the human gut microbiota, yet largely remain functionally uncharacterized [63]. In accordance with findings by Liu et al. [18], DHPS was desulfonated into sulfite and hydroxyacetone 

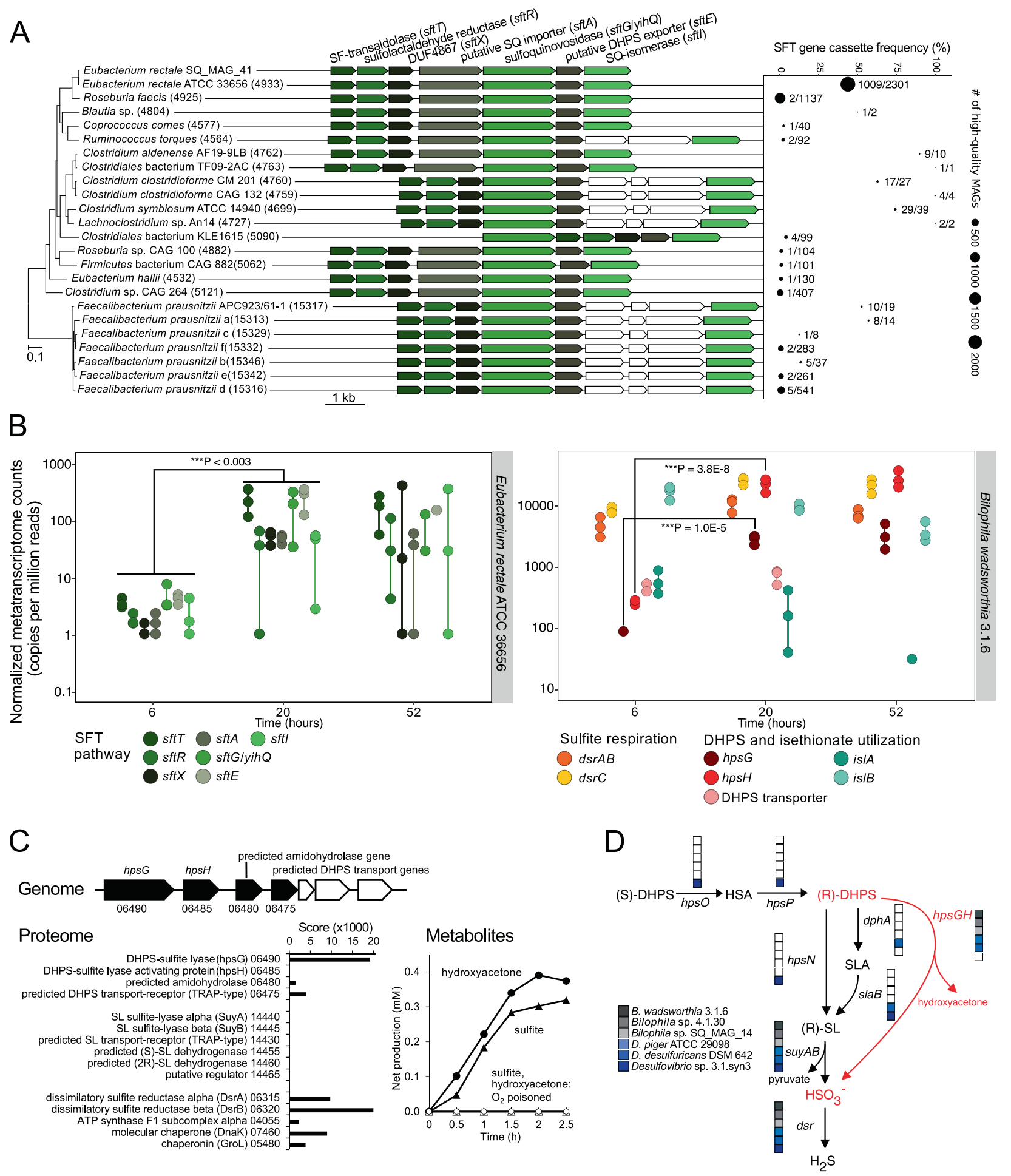

by HpsGH (Fig. S5E) in cell-free extracts of DHPSgrown $B$. wadsworthia cells, but only when assayed under strictly anoxic conditions (Fig. 2C), similar to the highly oxygen-sensitive IslA reaction [28, 62]. Furthermore, the pure culture excreted hydroxyacetone during DHPS degradation (Fig. S5A). While IslA cannot utilize DHPS as a substrate [28], HpsGH has a $~ 100$-fold preference for DHPS vs. isethionate [18]. B. wadsworthia thus uses HpsG, a glycyl radical $\mathrm{C}-\mathrm{S}$ bond-cleaving DHPS sulfite-lyase, to produce sulfite from DHPS intracellularly, which is then respired to $\mathrm{H}_{2} \mathrm{~S}$ via the DsrAB-DsrC-dissimilatory sulfite reductase pathway (Fig. 2D) [64, 65]. Collectively, we show that, in a human fecal microbiota, E. rectale and B. wadsworthia 
Fig. 2 Distribution, composition, and expression of genes involved in sulfoquinovose degradation by Eubacterium rectale and DHPS degradation by Bilophila wadsworthia. A Structure of the SQutilization gene cluster for SQ utilization via the SFT pathway in human gut bacteria and the E. rectale SQ_MAG_41 recovered from the microcosms. Genes depicted in white have no predicted function. The four core genes of the SFT pathway (in green shades) are present in 23 species-level MAG clusters (of 4,930 total clusters; each $>95 \%$ MAG ANI) that are Lachnospiraceae and Ruminococcaceae family members. MAG clusters are ordered based on the phylogeny of representative genomes. Numbers in parentheses denote MAG speciescluster IDs [45]. SQ_MAG_41 is a member of the E. rectale ATCC 33656 species cluster. SFT gene cassette frequency across each species-level MAG cluster with point labels indicating the number of MAGs containing the SQ-gene cassette with respect to the total number of MAGs in each species-level cluster. B Expression of pathways for SQ utilization by $E$. rectale (transcripts mapped to $E$. rectale ATCC 36656) (left) and DHPS utilization and sulfite respiration by $B$. wadsworthia (transcripts mapped to B. wadsworthia 3.1.6) (right) in the metatranscriptomes of triplicate $(n=3)$ fecal microcosms at 6,20 , and $52 \mathrm{~h}$ after amendment with $10 \mathrm{mM}$ SQ. Vertical lines connect non-overlapping replicate data points and asterisks indicate significant difference in expression level. DUF domain of unknown function, SF 6-deoxy-6-sulfofructose, SQ sulfoquinovose, DHPS 2,3dihydroxypropane-1-sulfonate, HSA 2-oxo-3-hydroxy-propane-1sulfonate, SLA 3-sulfolactaldehyde, SL 3-sulfolactate. C Structure of the DHPS utilization gene cluster in B. wadsworthia 3.1.6 and its inducibly expressed proteins (bar graph) during growth with DHPS as electron acceptor (numbers refer to RefSeq locus tag numbers; prefix HMPREF0179_RS) (Fig. S5C shows a comparison to growth with taurine, isethionate, and 3-sulfolactate as electron acceptors). Metabolite analysis (line graph) of cell-free extracts of DHPS-grown cells indicated cleavage of DHPS into sulfite and hydroxyacetone, if the reaction was performed under strictly anoxic conditions [28]; representative results $(n=5)$. D Presence/absence of DHPS-utilization pathway genes in $B$. wadsworthia genomes and other selected $d s r A B C$-encoding human gut Desulfovibrionaceae.

each employ recently discovered metabolic pathways to engage in interspecies DHPS transfer for joint degradation of plant diet-derived SQ to $\mathrm{H}_{2} \mathrm{~S}$.

\section{Active SQ fermentation and $\mathrm{H}_{2} \mathrm{~S}$ production across humans with various gut health states}

To explore how widely distributed and active SQDG/SQ metabolism is in the human gut, we analyzed the expression of the SFT pathway, and hpsGH, islAB, and $d s r A B C$ in the 1,090 stool metatranscriptomes from the Health Professionals Follow-Up Study (365 samples from 96 healthy men) [58] and an inflammatory bowel disease (IBD) study $(189,203$, and 333 samples from 27 healthy individuals, 28 with ulcerative colitis, and 50 with Crohn's disease, respectively) [60]. Consistent with previous reports $[58,60,66]$, glycolysis (from glucose and glucose-6-phosphate) and starch degradation were in the top $5 \%$ of 445 expressed microbial pathways across all datasets (Fig. 3A). We found that the SFT pathway was within the top third (136th) of all 445 expressed pathways and transcribed prevalently among individuals across all cohorts (177 out of 201 individuals). Further underlining the importance of SQ as a microbial nutrient in the gut, mean SFT pathway transcription was at a similar level to pathways for the usage of fucose (125th) and N-acetylneuraminate (149th) that are abundant components of glycoproteins and glycolipids in the colonic epithelium (Fig. 3A). Contrary to diet-derived $\mathrm{SQ}$, these host-derived sugars represent permanently available substrates for microorganisms in the gut [7]. Furthermore, we determined that the mean relative abundance of the SFT pathway was two orders of magnitude higher than the proteobacterial sulfo-EMP pathway (321st), which was actively expressed in only 28 samples (Fig. 3A). In contrast to what has been hypothesized [17], E. coli or other Enterobacteriaceae were only minor contributors to SQdegradation pathway expression, as assessed from fecal samples of these cohorts. We further found that in many individual samples $(51 \%)$ only a single bacterial species was responsible for more than 50\% of SFT pathway transcription. Eighteen putative SQ degraders of the families Lachnospiraceae and Ruminococcaceae (Firmicutes) expressed the SFT pathway (Fig. 3B). E. rectale, Faecalibacterium prausnitzii, Clostridium aldenense, Roseburia sp. AM16-25, and Clostridium clostridioforme contributed most to expression of the SFT pathway but E. rectale was the single most dominant species across both healthy individuals (Fig. 3B) and those with ulcerative colitis or Crohn's disease (276, 148, and 198 metatranscriptomes, respectively) (Fig. S7). The reasons for these speciesspecific SFT pathway expression patterns across individual fecal samples are unknown, but might depend on the dynamic intestinal concentrations of SQ and the possibly different kinetics of SQ-degrading enzymes of different microorganisms.

Genes for the DHPS sulfite-lyase ( $h p s G)$ and its activating enzyme $(h p s H)$ were also expressed in human stool (136 positive samples out of 1,090). Samples with detectable expression of the SFT pathway $(n=622)$ did have a higher proportion of samples showing expression of the DHPS pathway (15.6\% or 97 of 622 samples) when compared to those samples without detectable SFT pathway expression (8.3\% or 39 of 468 samples, Fig. 3C, Fig S7). However, this was not statistically significant and the asynchronous consumption of SQ and DHPS in the fecal microcosm incubations (Fig. 1A) suggests that expression of these pathways is also time-delayed. Furthermore, the isethionate sulfite-lyase genes (isl $A B$ ) that enable $B$. wadsworthia to utilize the sulfite from taurine and isethionate as an electron acceptor [28] were transcribed more prevalently (736 samples) and at a higher relative abundance when compared to hpsGH (Fig. 3C). Through meat consumption and microbial deconjugation of host-secreted taurocholic bile acids [2, 56, 67], taurine is a continuously available 

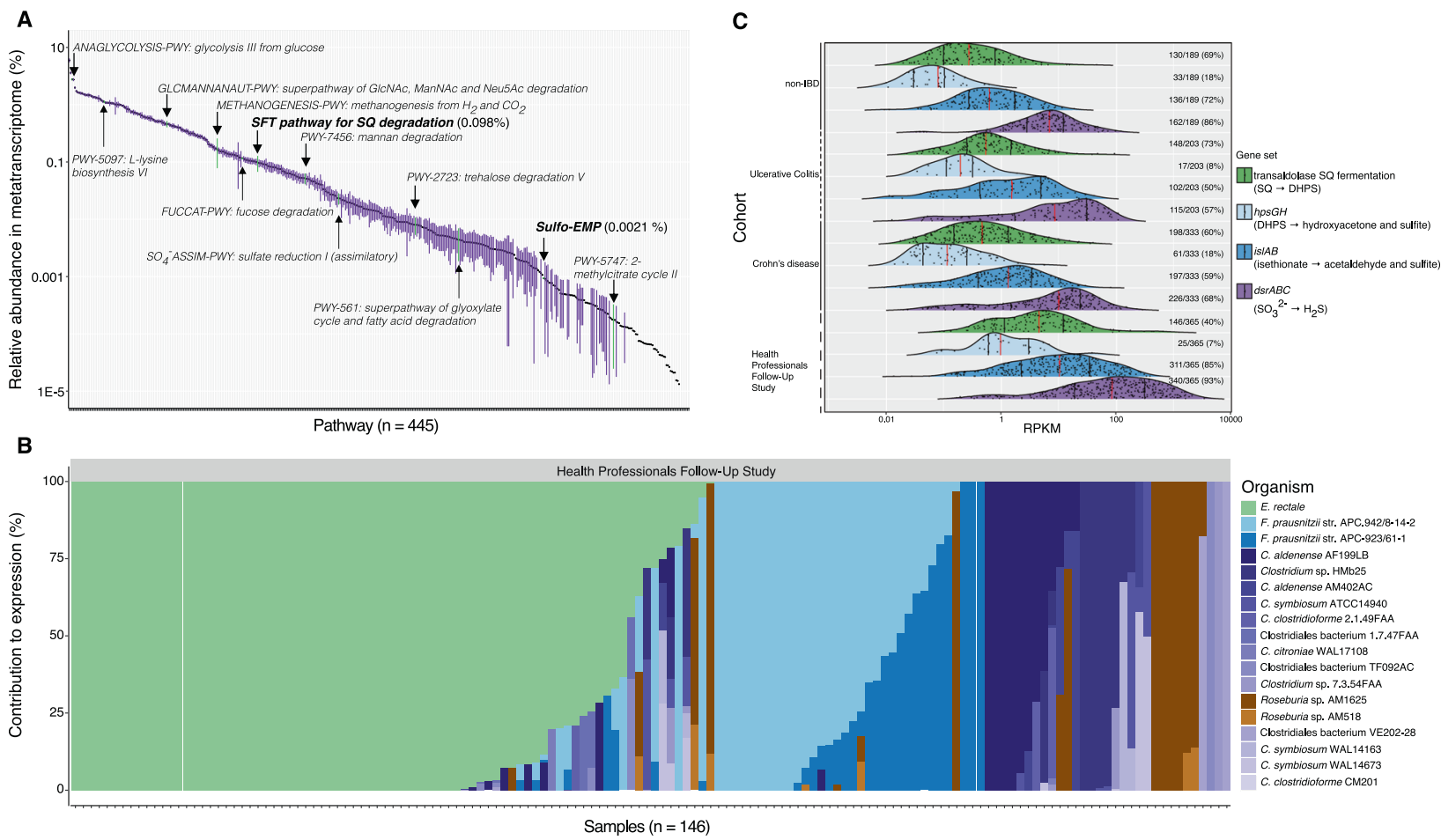

Fig. 3 Expression of microbial pathways for SQ fermentation and $\mathrm{H}_{2} \mathrm{~S}$ production are abundantly and frequently detected in human stool metatranscriptomes. A Ranked relative abundance of 445 identified pathways in 1,090 human stool metatranscriptomes from 201 individuals; each point is the mean relative abundance of a pathway and error bars correspond to the $95 \%$ confidence interval of the mean. B Relative \% contribution of various Firmicutes (depicted in various colors) to expression of the SFT pathway in the HPFS cohort $(n=146)$. Each bar corresponds to one sample. See "Materials and methods" for references. C Expression of the SFT pathway, DHPS and

isethionate utilization ( $h p s G H$ and $i s l A B$, respectively), and sulfite respiration $(d s r A B C)$ in 1,090 human stool metatranscriptomes from 201 individuals from 4 cohorts. The height and area of the ridgeline plots correspond to the distribution of expression. Each point corresponds to 1 sample, the red vertical lines depict the median, and the black lines correspond to the 25 th and 75 th percentiles. The number of samples with detectable expression within each group is indicated to the right of each plot. HPFS Health Professionals Follow-up Study, RPKM reads per kilobase per million reads, SQ sulfoquinovose, DHPS 2,3-dihydroxypropane-1-sulfonate.

substrate in the gut and thus could decouple the DHPSmediated physiological interaction of $B$. wadsworthia with the primary SQ fermenters. Transcription of the DsrABDsrC pathway for $\mathrm{H}_{2} \mathrm{~S}$ production was abundant and prevalent in the dataset (843 positive samples out of 1,090), and largely dominated by B. wadsworthia (Fig. 3C, Fig. S7). Notably, we did not detect significant differences in expression of SQ degradation and $\mathrm{H}_{2} \mathrm{~S}$ production pathways between cohorts of IBD patients and healthy individuals, which is likely because, as reported [66], stool samples were not selectively taken from patients with active IBD and thus, contrary to previous studies [68, 69], the observed species composition in stool was not significantly different between IBD and control cohorts. Overall, our reanalysis of stool metatranscriptomes from the four cohorts highlighted $B$. wadsworthia as a major sulfidogen and DHPS as an additional substrate for microbial $\mathrm{H}_{2} \mathrm{~S}$ production in the human gut. $B$. wadsworthia is specialized in utilizing diverse organosulfonates that are energetically more favorable compared to sulfate for sulfite-respiring bacteria $[17,28,70]$.

\section{Conclusions}

By uncovering the activities of genes for degradation of the plant-derived sulfonated monosaccharide SQ in the human gut microbiota, we have shown that SQ is an exclusive substrate for only a few gut microorganisms, particularly the abundant $E$. rectale. The concept of exclusive nutrient access [5] promises SQ dosage-dependent control over the abundances and activities of these bacteria that are generally associated with a positive impact on human health [3, 71]. We have also demonstrated that interspecies transfer of the SQ-degradation product DHPS is a previously unknown physiological link between a plant-based diet and $\mathrm{H}_{2} \mathrm{~S}$ production by the intestinal pathobiont B. wadsworthia, which uses DHPS in addition to taurine (Fig. 4). $\mathrm{H}_{2} \mathrm{~S}$ is an important intestinal metabolite that influences the activity of epithelial and microbial cells and has beneficial as well as detrimental effects on the colonic environment [24, 27]. The complex host-endogenous and microbial processes regulating intestinal $\mathrm{H}_{2} \mathrm{~S}$ homeostasis and how they are influenced by diet or health state are 


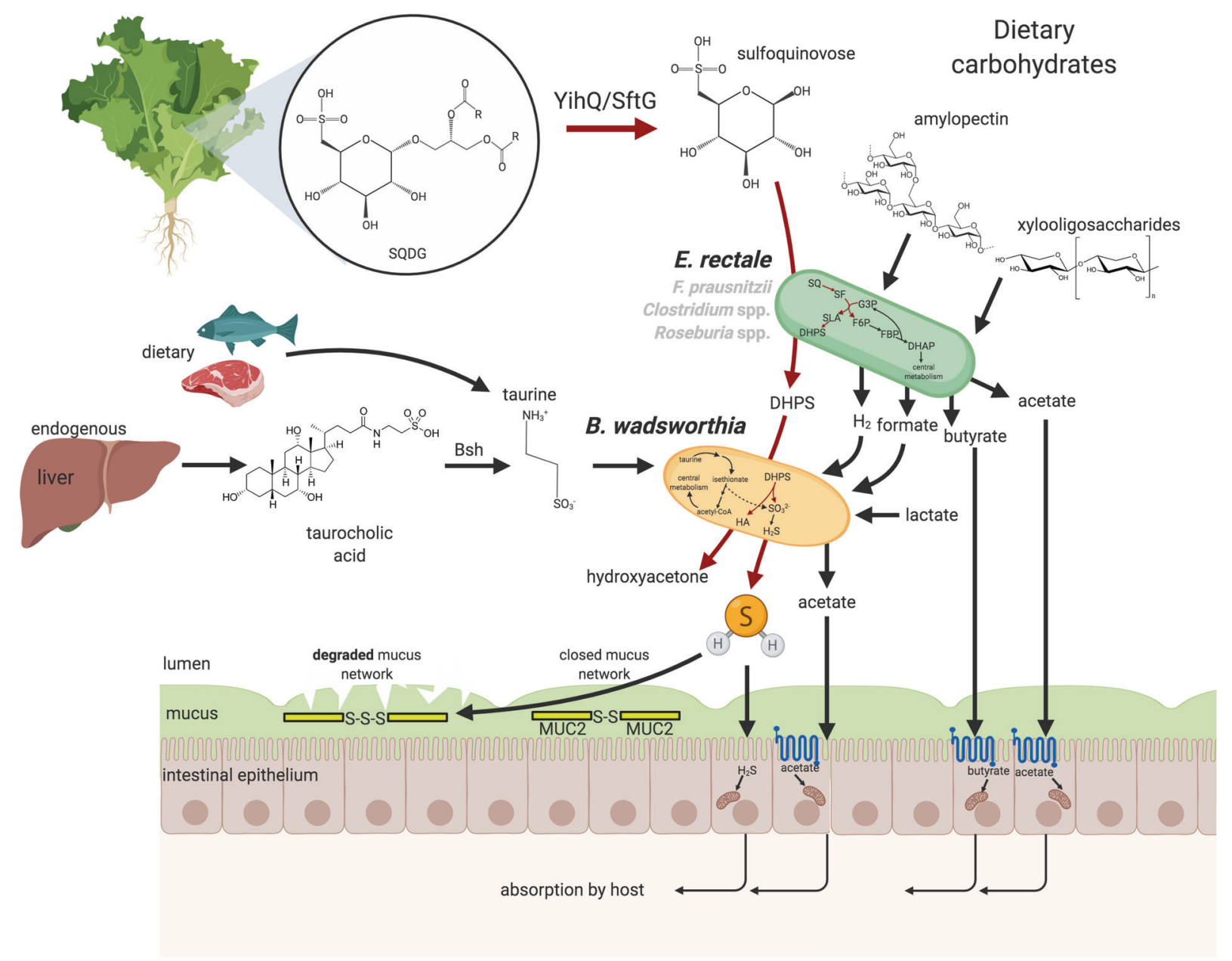

Fig. 4 Sulfur energy metabolism and physiological interaction scheme of $\boldsymbol{E}$. rectale and $\boldsymbol{B}$. wadsworthia in the human gut. SQDG present in green vegetables enters the intestine as a common component of the human diet. Sulfoquinovosidases [14], encoded by yih $Q /$ $s f t G$, cleave the glycosidic linkage of the lipid tail to liberate SQ, which is then in most individuals fermented mainly by E. rectale to DHPS, acetate, and likely also to formate or $\mathrm{H}_{2}$ and $\mathrm{CO}_{2}$. Further plant-derived compounds, such as amylopectin, xylooligosaccharides and other carbohydrates, may also be catabolized and fermented by E. rectale. Other Firmicutes with the SFT pathway (grey) such as members of the genera Faecalibacterium, Roseburia, and Clostridium can also function as primary SQ degraders in some individuals. Some Proteobacteria such as E. coli may catabolize SQ via the sulfo-EMP pathway. B. wadsworthia cleaves the C-S bond in DHPS via a DHPSsulfite lyase to produce sulfite for dissimilatory reduction to $\mathrm{H}_{2} \mathrm{~S}$ gas coupled to oxidation of, e.g., formate, lactate, and hydrogen, while hydroxyacetone and also acetate are excreted. In few individuals, also Desulfovibrio piger uses DHPS as substrate for anaerobic sulfite respiration. Taurine is present in dietary meat/fish and is liberated from

insufficiently defined [20, 27]. Our work emphasizes the roles of microbial cooperation and sulfur metabolism in the human intestinal tract and uncovers new physiological and genetic features of prevalent microbiota members that will inform dietary and drug-based therapies [27] for targeted modification of microbiota membership and $\mathrm{H}_{2} \mathrm{~S}$ levels in intestinal disease. host-secreted taurocholic acids by microbial bile salt hydrolases (encoded by $b s h$ ), and thus far was considered the sole major source of sulfonate-sulfur for respiration and $\mathrm{H}_{2} \mathrm{~S}$ production by $\mathrm{B}$. wadsworthia in the human intestine. $\mathrm{H}_{2} \mathrm{~S}$ and short-chain fatty acids are utilized by colonic epithelial cells as energy sources, act as signaling molecules, and are absorbed and further distributed by the host via the bloodstream [72-74]. Excessive concentrations of $\mathrm{H}_{2} \mathrm{~S}$ gas can break the mucus layer in the colon by reduction of Muc2-mucin disulfide bonds to trisulfides [24], thereby allowing bacteria to penetrate the mucus layer and interact with the host epithelial lining. Arrows colored in black represent previously described metabolic pathways, while those in dark red represent pathways revealed in this study. SQDG sulfoquinovosyl diacylglycerol, SQ sulfoquinovose (6-deoxy-6-sulfoglucose), SF 6-deoxy-6-sulfofructose, G3P glyceraldehyde-3-phosphate, F6P fructose-6-phosphate, FBP fructose-1,6-bisphosphate, DHAP dihydroxyacetone phosphate, SLA 3-sulfolactaldehyde, DHPS 2,3dihydroxypropane-1-sulfonate, HA hydroxyacetone. This figure was created using Biorender.com.

\section{Data availability}

All sequence data generated in this project is available at NCBI under BioProject PRJNA593787.

Acknowledgements We acknowledge support from the BiomedicalSequencing Facility (https://www.biomedical-sequencing.org) for 
metagenome and metatranscriptome sequencing, from the Konstanz Proteomics Centre (https://www.biologie.uni-konstanz.de/proteomicscentre/), and from Patricia Wolf for optimizing hybridization conditions for the BWA829 FISH probe during the 2016 International FISH Course at the Division of Microbial Ecology, University of Vienna, Austria. We are thankful to Frank Maixner for initiation of collaboration and to the DOME gut group members in Vienna as well as to our colleagues at University of Konstanz for fruitful discussions and support. This work was financially supported by the Austrian Science Fund (FWF; project grants I2320-B22 and DOC 69-B), the Deutsche Forschungsgemeinschaft (DFG; grants SCHL1936/3-4; KoRS-CB), and the European Union (MSCA-IF project 796687 - H2Gut).

Author contributions BTH, DK, DS, and AL conceived the study. $\mathrm{BTH}, \mathrm{DK}, \mathrm{JL}, \mathrm{AGB}, \mathrm{AF}, \mathrm{KD}, \mathrm{BF}, \mathrm{CWH}$, and $\mathrm{NK}$ performed experiments and analyzed data. TR and NS provided bioinformatic support. DS and AL helped with experimental design and data interpretation, and DS helped with writing. BTH, DK, and AL wrote the article. All authors discussed the results and revised the manuscript.

\section{Compliance with ethical standards}

Conflict of interest The authors declare no competing interests.

Publisher's note Springer Nature remains neutral with regard to jurisdictional claims in published maps and institutional affiliations.

Open Access This article is licensed under a Creative Commons Attribution 4.0 International License, which permits use, sharing, adaptation, distribution and reproduction in any medium or format, as long as you give appropriate credit to the original author(s) and the source, provide a link to the Creative Commons license, and indicate if changes were made. The images or other third party material in this article are included in the article's Creative Commons license, unless indicated otherwise in a credit line to the material. If material is not included in the article's Creative Commons license and your intended use is not permitted by statutory regulation or exceeds the permitted use, you will need to obtain permission directly from the copyright holder. To view a copy of this license, visit http://creativecommons. org/licenses/by/4.0/.

\section{References}

1. Thaiss CA, Itav S, Rothschild D, Meijer MT, Levy M, Moresi C, et al. Persistent microbiome alterations modulate the rate of postdieting weight regain. Nature. 2016;540:544-51.

2. David LA, Maurice CF, Carmody RN, Gootenberg DB, Button JE, Wolfe BE, et al. Diet rapidly and reproducibly alters the human gut microbiome. Nature. 2014;505:559-63.

3. Zeevi D, Korem T, Zmora N, Israeli D, Rothschild D, Weinberger A, et al. Personalized nutrition by prediction of glycemic responses. Cell. 2015;163:1079-94.

4. Barabási A-L, Menichetti G, Loscalzo J. The unmapped chemical complexity of our diet. Nat Food. 2019;1:33-37.

5. Shepherd ES, DeLoache WC, Pruss KM, Whitaker WR, Sonnenburg JL. An exclusive metabolic niche enables strain engraftment in the gut microbiota. Nature. 2018;557:434-8.

6. Hehemann J-H, Correc G, Barbeyron T, Helbert W, Czjzek M, Michel G. Transfer of carbohydrate-active enzymes from marine bacteria to Japanese gut microbiota. Nature. 2010;464:908-12.

7. Bell A, Brunt J, Crost E, Vaux L, Nepravishta R, Owen CD, et al. Elucidation of a sialic acid metabolism pathway in mucus- foraging Ruminococcus gnavus unravels mechanisms of bacterial adaptation to the gut. Nat Microbiol. 2019;4:2393-404.

8. Heintz-Buschart A, Wilmes P. Human gut microbiome: function matters. Trends Microbiol. 2018;26:563-74.

9. Goddard-Borger ED, Williams SJ. Sulfoquinovose in the biosphere: occurrence, metabolism and functions. Biochem J. 2017;474:827-49.

10. Benning C. Biosynthesis and function of the sulfolipid sulfoquinovosyl diacylglycerol. Annu Rev Plant Physiol Plant Mol Biol. 1998;49:53-75.

11. Gupta SD, Sastry PS. Metabolism of the plant sulfolipid-sulfoquinovosyldiacylglycerol: degradation in animal tissues. Arch Biochem Biophys. 1987;259:510-9.

12. Denger K, Weiss M, Felux A-K, Schneider A, Mayer C, Spiteller D, et al. Sulphoglycolysis in Escherichia coli $\mathrm{K}-12$ closes a gap in the biogeochemical sulphur cycle. Nature. 2014;507:114-7.

13. Felux A-K, Spiteller D, Klebensberger J, Schleheck D. EntnerDoudoroff pathway for sulfoquinovose degradation in Pseudomonas putida SQ1. Proc Natl Acad Sci USA. 2015;112: E4298-305.

14. Abayakoon P, Jin Y, Lingford JP, Petricevic M, John A, Ryan E, et al. Structural and biochemical insights into the function and evolution of sulfoquinovosidases. ACS Cent Sci. 2018;4:1266-73.

15. Frommeyer B, Fiedler AW, Oehler SR, Hanson BT, Loy A, Franchini $\mathrm{P}$, et al. Environmental and intestinal phylum Firmicutes bacteria metabolize the plant sugar sulfoquinovose via a 6-deoxy6-sulfofructose transaldolase pathway. iScience 2020;23:101510.

16. Liu Y, Wei Y, Zhou Y, Ang EL, Zhao H, Zhang Y. A transaldolase-dependent sulfoglycolysis pathway in Bacillus megaterium DSM 1804. Biochem. Biophys. Res. Commun. 2020;533:1109-14.

17. Burrichter A, Denger K, Franchini P, Huhn T, Müller N, Spiteller $\mathrm{D}$, et al. Anaerobic degradation of the plant sugar sulfoquinovose concomitant with $\mathrm{H}_{2} \mathrm{~S}$ production: Escherichia coli $\mathrm{K}-12$ and Desulfovibrio sp. strain DF1 as Co-culture Model. Front Microbiol. 2018;9:2792.

18. Liu J, Wei Y, Lin L, Teng L, Yin J, Lu Q, et al. Two radicaldependent mechanisms for anaerobic degradation of the globally abundant organosulfur compound dihydroxypropanesulfonate. Proc Natl Acad Sci USA. 2020;117:15599-608.

19. Kabil O, Vitvitsky V, Banerjee R. Sulfur as a signaling nutrient through hydrogen sulfide. Annu Rev Nutr. 2014;34:171-205.

20. Carbonero F, Benefiel AC, Alizadeh-Ghamsari AH, Gaskins HR. Microbial pathways in colonic sulfur metabolism and links with health and disease. Front Physiol. 2012;3:448.

21. Barton LL, Ritz NL, Fauque GD, Lin HC. Sulfur cycling and the intestinal microbiome. Dig Dis Sci. 2017;62:2241-57.

22. Banerjee R. Hydrogen sulfide: redox metabolism and signaling. Antioxid Redox Signal. 2011;15:339-41.

23. Olson KR, Straub KD. The role of hydrogen sulfide in evolution and the evolution of hydrogen sulfide in metabolism and signaling. Physiology. 2016;31:60-72.

24. Ijssennagger N, Belzer C, Hooiveld GJ, Dekker J, van Mil SWC, Müller M, et al. Gut microbiota facilitates dietary heme-induced epithelial hyperproliferation by opening the mucus barrier in colon. Proc Natl Acad Sci USA. 2015;112:10038-43.

25. Beauchamp RO Jr, Bus JS, Popp JA, Boreiko CJ, Andjelkovich DA. A critical review of the literature on hydrogen sulfide toxicity. Crit Rev Toxicol. 1984;13:25-97.

26. Devkota S, Wang Y, Musch MW, Leone V, Fehlner-Peach H, Nadimpalli A, et al. Dietary-fat-induced taurocholic acid promotes pathobiont expansion and colitis in I110-/- mice. Nature. $2012 ; 487: 104-8$. 
27. Wallace JL, Motta J-P, Buret AG. Hydrogen sulfide: an agent of stability at the microbiome-mucosa interface. Am J Physiol Gastrointest Liver Physiol. 2018;314:G143-G149.

28. Peck SC, Denger K, Burrichter A, Irwin SM, Balskus EP, Schleheck D. A glycyl radical enzyme enables hydrogen sulfide production by the human intestinal bacterium. Proc Natl Acad Sci USA. 2019;116:3171-6.

29. Cline JD. Spectrophotometric determination of hydrogen sulfide in natural waters. Limnol Oceanogr. 1969;14:454-8.

30. Ludwig W, Strunk O, Westram R, Richter L, Meier H, Yadhukumar, et al. ARB: a software environment for sequence data. Nucleic Acids Res. 2004;32:1363-71.

31. Daims H, Stoecker K, Wagner M. Fluorescence in situ hybridization for the detection of prokaryotes. In: Osborn A, Smith C, editors. Advanced Methods in Molecular Microbial Ecology. 2005. Abingdon, UK: Bios-Garland. p. 213-39.

32. Berry D, Mader E, Lee TK, Woebken D, Wang Y, Zhu D, et al. Tracking heavy water $\left(\mathrm{D}_{2} \mathrm{O}\right)$ incorporation for identifying and sorting active microbial cells. Proc Natl Acad Sci USA. 2015;112: E194-203.

33. Griffiths RI, Whiteley AS, O'Donnell AG, Bailey MJ. Rapid method for coextraction of DNA and RNA from natural environments for analysis of ribosomal DNA- and rRNA-based microbial community composition. Appl Environ Microbiol. 2000;66:5488-91.

34. Herbold CW, Pelikan C, Kuzyk O, Hausmann B, Angel R, Berry $\mathrm{D}$, et al. A flexible and economical barcoding approach for highly multiplexed amplicon sequencing of diverse target genes. Front Microbiol. 2015;6:731.

35. Pelikan C, Herbold CW, Hausmann B, Müller AL, Pester M, Loy A. Diversity analysis of sulfite- and sulfate-reducing microorganisms by multiplex $d s r A$ and $d s r B$ amplicon sequencing using new primers and mock community-optimized bioinformatics. Environ Microbiol. 2016;18:2994-3009.

36. Nurk S, Meleshko D, Korobeynikov A, Pevzner PA. metaSPAdes: a new versatile metagenomic assembler. Genome Res. 2017;27:824-34.

37. Kang DD, Froula J, Egan R, Wang Z. MetaBAT, an efficient tool for accurately reconstructing single genomes from complex microbial communities. PeerJ. 2015;3:e1165.

38. Parks DH, Imelfort M, Skennerton CT, Hugenholtz P, Tyson GW. CheckM: assessing the quality of microbial genomes recovered from isolates, single cells, and metagenomes. Genome Res. 2015;25:1043-55.

39. Olm MR, Brown CT, Brooks B, Banfield JF. dRep: a tool for fast and accurate genomic comparisons that enables improved genome recovery from metagenomes through de-replication. ISME J. 2017;11:2864-8.

40. Parks DH, Chuvochina M, Waite DW, Rinke C, Skarshewski A, Chaumeil P-A, et al. A standardized bacterial taxonomy based on genome phylogeny substantially revises the tree of life. Nat Biotechnol. 2018;36:996-1004.

41. Jain C, Rodriguez-R LM, Phillippy AM, Konstantinidis KT, Aluru S. High throughput ANI analysis of $90 \mathrm{~K}$ prokaryotic genomes reveals clear species boundaries. Nat Commun. 2018;9:5114.

42. Liao Y, Smyth GK, Shi W. featureCounts: an efficient general purpose program for assigning sequence reads to genomic features. Bioinformatics. 2014;30:923-30.

43. Potter SC, Luciani A, Eddy SR, Park Y, Lopez R, Finn RD. HMMER web server: 2018 update. Nucleic Acids Res. 2018;46: W200-W204.

44. Speciale G, Jin Y, Davies GJ, Williams SJ, Goddard-Borger ED. YihQ is a sulfoquinovosidase that cleaves sulfoquinovosyl diacylglyceride sulfolipids. Nat Chem Biol. 2016;12:215-7.
45. Pasolli E, Asnicar F, Manara S, Zolfo M, Karcher N, Armanini F, et al. Extensive unexplored human microbiome diversity revealed by over 150,000 genomes from metagenomes spanning age, geography, and lifestyle. Cell. 2019;176:649-62.e20.

46. Franzosa EA, McIver LJ, Rahnavard G, Thompson LR, Schirmer $\mathrm{M}$, Weingart G, et al. Species-level functional profiling of metagenomes and metatranscriptomes. Nat Methods. 2018;15:962-8.

47. Truong DT, Franzosa EA, Tickle TL, Scholz M, Weingart G, Pasolli E, et al. MetaPhlAn2 for enhanced metagenomic taxonomic profiling. Nat Methods. 2015;12:902-3.

48. Kurtz ZD, Müller CL, Miraldi ER, Littman DR, Blaser MJ, Bonneau RA. Sparse and compositionally robust inference of microbial ecological networks. PLoS Comput Biol. 2015;11: e1004226.

49. Shannon P, Markiel A, Ozier O, Baliga NS, Wang JT, Ramage D, et al. Cytoscape: a software environment for integrated models of biomolecular interaction networks. Genome Res. 2003;13:2498-504.

50. Nepusz T, Yu H, Paccanaro A. Detecting overlapping protein complexes in protein-protein interaction networks. Nat Methods. 2012;9:471-2.

51. Lee MD. GToTree: a user-friendly workflow for phylogenomics. Bioinformatics. 2019;35:4162-4.

52. Katoh K, Standley DM. MAFFT multiple sequence alignment software version 7: improvements in performance and usability. Mol Biol Evol. 2013;30:772-80.

53. Edgar RC. Search and clustering orders of magnitude faster than BLAST. Bioinformatics. 2010;26:2460-1.

54. Kraal L, Abubucker S, Kota K, Fischbach MA, Mitreva M. The prevalence of species and strains in the human microbiome: a resource for experimental efforts. PLoS ONE. 2014;9:e97279.

55. Karcher N, Pasolli E, Asnicar F, Huang KD, Tett A, Manara S, et al. Analysis of 1321 Eubacterium rectale genomes from metagenomes uncovers complex phylogeographic population structure and subspecies functional adaptations. Genome Biol. 2020;21:138.

56. Ridlon JM, Wolf PG, Gaskins HR. Taurocholic acid metabolism by gut microbes and colon cancer. Gut Microbes. 2016;7:201-15.

57. Wang R. Physiological implications of hydrogen sulfide: a whiff exploration that blossomed. Physiol Rev. 2012;92:791-896.

58. Abu-Ali GS, Mehta RS, Lloyd-Price J, Mallick H, Branck T, Ivey $\mathrm{KL}$, et al. Metatranscriptome of human faecal microbial communities in a cohort of adult men. Nat Microbiol. 2018;3:356-66.

59. Mehta RS, Abu-Ali GS, Drew DA, Lloyd-Price J, Subramanian A, Lochhead P, et al. Stability of the human faecal microbiome in a cohort of adult men. Nat Microbiol. 2018;3:347-55.

60. Schirmer M, Franzosa EA, Lloyd-Price J, McIver LJ, Schwager R, Poon TW, et al. Dynamics of metatranscription in the inflammatory bowel disease gut microbiome. Nat Microbiol. 2018;3:337-46.

61. Mayer J, Huhn T, Habeck M, Denger K, Hollemeyer K, Cook AM. 2,3-Dihydroxypropane-1-sulfonate degraded by Cupriavidus pinatubonensis JMP134: purification of dihydroxypropanesulfonate 3dehydrogenase. Microbiology. 2010;156:1556-64.

62. Xing M, Wei Y, Zhou Y, Zhang J, Lin L, Hu Y, et al. Radicalmediated $\mathrm{C}-\mathrm{S}$ bond cleavage in $\mathrm{C} 2$ sulfonate degradation by anaerobic bacteria. Nat. Commun. 2019;10:1609.

63. Levin BJ, Balskus EP. Discovering radical-dependent enzymes in the human gut microbiota. Curr Opin Chem Biol. 2018;47:86-93.

64. Laue H, Friedrich M, Ruff J, Cook AM. Dissimilatory sulfite reductase (desulfoviridin) of the taurine-degrading, non-sulfatereducing bacterium Bilophila wadsworthia RZATAU contains a fused DsrB-DsrD subunit. J Bacteriol. 2001;183:1727-33.

65. Santos AA, Venceslau SS, Grein F, Leavitt WD, Dahl C, Johnston DT, et al. A protein trisulfide couples dissimilatory 
sulfate reduction to energy conservation. Science. 2015; 350:1541-5.

66. Lloyd-Price J, Arze C, Ananthakrishnan AN, Schirmer M, AvilaPacheco J, Poon TW, et al. Multi-omics of the gut microbial ecosystem in inflammatory bowel diseases. Nature. 2019;569:655-62.

67. Huxtable RJ, Franconi F, Giotti A (eds). The biology of taurine: methods and mechanisms. New York: Plenum Press; 1987.

68. Gevers D, Kugathasan S, Denson LA, Vázquez-Baeza Y, Van Treuren W, Ren B, et al. The treatment-naive microbiome in newonset Crohn's disease. Cell Host Microbe. 2014;15:382-92.

69. Morgan XC, Tickle TL, Sokol H, Gevers D, Devaney KL, Ward DV, et al. Dysfunction of the intestinal microbiome in inflammatory bowel disease and treatment. Genome Biol. 2012;13:R79.
70. Rey FE, Gonzalez MD, Cheng J, Wu M, Ahern PP, Gordon JI. Metabolic niche of a prominent sulfate-reducing human gut bacterium. Proc Natl Acad Sci USA. 2013;110:13582-7.

71. Tian Y, Wang K, Ji G. Faecalibacterium Prausnitzii prevents tumorigenesis in a model of colitis-associated colorectal cancer. Gastroenterology. 2017;152:S354-5

72. Pomare EW, Branch WJ, Cummings JH. Carbohydrate fermentation in the human colon and its relation to acetate concentrations in venous blood. J Clin Investig. 1985;75:1448-54.

73. Zambell KL, Fitch MD, Fleming SE. Acetate and butyrate are the major substrates for de novo lipogenesis in rat colonic epithelial cells. J Nutr. 2003;133:3509-15.

74. Fu M, Zhang W, Wu L, Yang G, Li H, Wang R. Hydrogen sulfide $\left(\mathrm{H}_{2} \mathrm{~S}\right)$ metabolism in mitochondria and its regulatory role in energy production. Proc Natl Acad Sci USA. 2012;109:2943-8. 